\title{
ON THE EXISTENCE OF STABLE PERIODIC SOLUTIONS OF DIFFERENTIAL EQUATIONS OF DUFFING TYPE
}

\author{
A. C. LAZER AND P. J. MCKENNA \\ (Communicated by Kenneth R. Meyer)
}

\begin{abstract}
We consider a second-order differential equation periodic in $t$ with period $T>0$ and with linear damping. Bounds are given for the derivative of the restoring force which will guarantee the existence and uniqueness of a $T$-periodic solution such that the unique $T$-periodic solution is asymptotically stable. These conditions also rule out the existence of additional periodic solutions which are subharmonics of order 2 .
\end{abstract}

In this note we consider periodic solutions of the differential equation

$$
u^{\prime \prime}+k u^{\prime}+g(t, u)=0
$$

where $k>0$ is a constant, $g$ and its partial derivative with respect to the second variable, denoted by $D_{2} g$, are continuous, and $g$ is $T$-periodic in $t$ for some $T>0$. Our goal is to find conditions of the form

$$
a \leq D_{2} g(t, \xi) \leq b
$$

for $(t, \xi) \in \mathbf{R}^{2}$ which will guarantee the existence and uniqueness of a $T$ periodic solution $u_{0}$ which is locally, exponentially, asymptotically stable, i.e. such that there exist constants $C>0$ and $\alpha>0$ such that if $u$ is another solution with $\left|u(0)-u_{0}(0)\right|$ and $\left|u^{\prime}(0)-u_{0}^{\prime}(0)\right|$ sufficiently small, then $\mid u(t)-$ $u_{0}(t)\left|\leq C d e^{-\alpha t}, \quad\right| u^{\prime}(t)-u_{0}^{\prime}(t) \mid \leq C d e^{-\alpha t}$ for all $t>0$, where $d=\mid u(0)-$ $u_{0}(0)|+| u^{\prime}(0)-u_{0}^{\prime}(0) \mid$.

The existence and uniqueness part of this problem has been considered in several papers when $k=0$. In this case, if there exists an integer $N \geq 0$ such that $4 \pi^{2} N^{2} / T^{2}<a \leq b<4 \pi^{2}(N+1)^{2} / T^{2}$ and (2) holds, then there exists a unique $T$-periodic solution of (1). This follows from work of Loud [6], under the additional assumption that a certain symmetry condition holds, and

Received by the editors May 20, 1989 and, in revised form, August 8, 1989.

1980 Mathematics Subject Classification (1985 Revision). Primary 34C25, 34D20.

Key words and phrases. Contraction mapping theorem, fundamental matrix, characteristic multipliers, asymptotically, exponentially stable.

The first author's research was supported by NSF grant DMS-8722593 and State of Ohio Academic Challenge Grant while visiting University of Cincinatti.

The second author's research was supported by NSF grant DMS-8722532. 
from work of Leach [5] in the general case. The Loud-Leach result can also be obtained from a theorem concerning Hammerstein integral equations due to Dolph in [4].

In the case of a second-order linear differential equation, the Loud-Leach result seems to be implicit in the works of Borg [2] and Wallach [10] which predate that of Dolph (see also [7, Chap. 5]).

Recently, there have appeared some papers in which conditions on $D_{2} g$ have been given which imply the existence of periodic solutions of (1) with $k \neq 0$. (See, for example, [9, 12-16].) However, except for the results of [9], which we briefly describe below, the stability of these periodic solutions has not been considered.

Our main results, Theorems 2 and 3 below, say that if the range of $D_{2} g$ is in an interval contained in a closed disk in the complex plane which contains no (complex) eigenvalue $\lambda$ of the problem

$$
\begin{gathered}
-u^{\prime \prime}-k u^{\prime}=\lambda u, \\
u(0)=u(2 T), \quad u^{\prime}(0)=u^{\prime}(2 T),
\end{gathered}
$$

then (1) has a unique $T$-periodic solution which is locally, exponentially, asymptotically stable. Note that the boundary conditions correspond to $2 T$-periodicity rather than $T$-periodicity!)

The proof of the following result is a modification of an argument given by Williams in [11]. Williams considered elliptic boundary value problems in which the differential operator is normal.

A preliminary lemma. Let $k \in \mathbf{R}$ and let $g(t, \xi)$ be defined and continuous for $(t, \xi) \in \mathbf{R}^{2}$, have a piecewise continuous derivative with respect to $\xi$, and be $T$-periodic in $t$ where $T>0$. If there exist numbers $a$ and $b$ such that the condition (2) holds for all $(t, \xi) \in \mathbf{R}^{2}$ and such that there exists a closed disk $B$ in the complex plane centered at $\gamma=(a+b) / 2$ of radius $r>(b-a) / 2$ such that

$$
\frac{4 \pi^{2} m^{2}}{T^{2}}-\frac{2 \pi i m}{T} k \notin B
$$

for all $m=0, \pm 1, \pm 2, \ldots$, then there exists a unique $T$-periodic solution of (1).

Proof. Let $L$ be the linear differential operator defined by $L u=-u^{\prime \prime}-k u^{\prime}-\gamma u$ and let $\mathbf{E}$ denote the Hilbert space consisting of complex valued $T$-periodic functions defined on $(-\infty, \infty)$ whose restrictions to the interval $[0, T]$ belong to $L^{2}[0, T]$ with the usual $L^{2}[0, T]$-inner product. If $h \in \mathbf{E}$, then there exists a unique $T$-periodic function $u$ such that $u$ is of class $C^{1}, u^{\prime}$ is absolutely continuous, $u " \in \mathbf{E}$ and $L u=h$. In fact, if in terms of the orthogonal basis $\left\{e^{2 \pi i m t / T} \mid m=0, \pm 1, \pm 2, \ldots\right\}$

$$
h(t)=\sum_{-\infty}^{\infty} c_{m} e^{2 \pi i m t / T}, \quad(\text { in } \mathbf{E})
$$


then

$$
u(t)=\sum_{m=-\infty}^{\infty} c_{m}\left(\frac{4 \pi^{2} m^{2}}{T^{2}}-\frac{2 \pi i m k}{T}-\gamma\right)^{-1} e^{2 \pi i m t / T}
$$

If we denote $u$ by $K h$, then $K$ may be viewed as a compact linear mapping from $\mathbf{E}$ into $\mathbf{E}$. Moreover, because of the inequality

$$
\left|4 \pi^{2} m^{2} / T^{2}-2 \pi i m k / T-\gamma\right|>r
$$

which follows from (3) for all $m=0, \pm 1, \pm 2, \ldots$, it follows from (4) and (5) that

$$
\|K h\| \leq(1 / r)\|h\|
$$

for all $h \in \mathbf{E}$, where \|\| is the norm in $\mathbf{E}$.

If we let $H$ denote real Hilbert space consisting of all real-valued functions in $E$ (so that $c_{-m}=\bar{c}_{m}$ in (4) if $h \in H$ ), then $K(H) \subset H$.

We have that $u$ is a $T$-periodic solution of (1) if and only if $u \in H$ and

$$
-u^{\prime \prime}-k u^{\prime}-\gamma u=L u=g(t, u)-\gamma u
$$

and therefore,

$$
u=K G(u) \equiv F(u),
$$

where $G: H \rightarrow H$ is the Nemytskii mapping defined by

$$
u \rightarrow g(t, u)-\gamma u \text {. }
$$

From (2) it follows that

$$
\left\|G\left(u_{1}\right)-G\left(u_{2}\right)\right\| \leq\left(\frac{b-a}{2}\right)\left\|u_{1}-u_{2}\right\|
$$

for all $u_{1}, u_{2} \in H$ and therefore,

$$
\left\|F\left(u_{1}\right)-F\left(u_{2}\right)\right\| \leq c\left\|u_{1}-u_{2}\right\|
$$

where $c=(b-a) / 2 r<1$. The assertion of the lemma now follows from the contraction mapping theorem.

A short proof of a system form of the Loud-Leach result based on this type of argument is given in [8].

Before proving our main result on (1), we prove a result concerning the characteristic multipliers of a linear system.

Theorem 1. Let $p(t)$ be a continuous $T$-periodic function and let $X(t)$ be the fundamental matrix for the linear system

$$
x^{\prime}(t)=A(t) x(t)
$$

where $x=\operatorname{col}\left(x_{1}, x_{2}\right)$,

$$
A(t)=\left(\begin{array}{cc}
0 & 1 \\
-p(t) & -k
\end{array}\right)
$$


and $k>0$ is constant. If $t$ here exist numbers $a$ and $b$ such that for all $t \in \mathbf{R}$

$$
a \leq p(t) \leq b
$$

for all $t$, and such that there exists a closed disk $B$ in the complex plane centered at $\gamma=(a+b) / 2$ of radius $r>(b-a) / 2$ such that

$$
\frac{\pi^{2} m^{2}}{T^{2}}-\frac{\pi i m k}{T} \notin B
$$

for all $m=0, \pm 1, \pm 2, \ldots$, then the eigenvalues $\alpha_{1}$ and $\alpha_{2}$ of $X(T)$ satisfy $\left|\alpha_{j}\right|<1$ for $j=1,2$.

Proof. For each $s$ with $0 \leq s \leq 1$ we have

$$
a \leq(1-s) \gamma+s p(t) \leq b
$$

for all $t$. Therefore, from the preliminary lemma and (9), for each $s \in[0,1]$, there exists a unique periodic solution of period $2 T$ of the linear homogeneous differential equation

$$
u^{\prime \prime}+k u^{\prime}+[(1-s) \gamma+s p(t)] u=0 .
$$

But $u \equiv 0$ is such a solution. Therefore, under the hypotheses of the theorem, there exists no nontrivial $2 T$-periodic solution of (11) for all $s \in[0,1]$. If

$$
B(s, t)=\left(\begin{array}{cc}
0 & 1 \\
-(1-s) \gamma-s p(t) & -k
\end{array}\right)
$$

then $y=\operatorname{col}\left(y_{1}, y_{2}\right)$ is a $2 T$-periodic solution of

$$
y^{\prime}(t)=B(t, s) y(t)
$$

if and only if $y_{1}=u$ and $y_{2}=u^{\prime}$ where $u$ is a nontrivial $2 T$-periodic solution of (11). Therefore, the system (12) has no nontrivial $2 T$-periodic solution for $0 \leq s \leq 1$.

Let $Y(t, s)$ denote the fundamental matrix associated with the system (12) for $0 \leq s \leq 1$. We have that

$$
Y^{\prime}(t, s)=B(t, s) Y(t, s), \quad Y(0, s)=I,
$$

where $I$ is the $2 \times 2$ identity. If for some $v \in \mathbf{R}^{2}$

$$
Y(T, s) v=-v,
$$

then $y(t) \equiv Y(t, s) v$ is a solution of the system (12) which satisfies $y(t)=$ $-y(0)$, so by virtue of the fact that both $-y(t)$ and $y(t+T)$ are solutions of (12) which are equal at $t=0$ and the uniqueness theorem, it follows that $y(t+T) \equiv-y(t)$. Hence, $y(t+2 T)=y(t)$ so $y(t) \equiv 0$ and therefore $v=0$.

It follows that -1 is not an eigenvalue of $Y(T, s)$ for all $s \in[0,1]$.

A similar argument shows that if $Y(T, s) v=v$, then $y(t) \equiv Y(t, s) v$ is a $T$-periodic solution and hence a $2 T$-periodic solution of (12). Hence for $0 \leq s \leq 1,1$ is not an eigenvalue of $Y(s, T)$. 
For each $s$ with $0 \leq s \leq 1$, let $\mu_{1}(s)$ and $\mu_{2}(s)$ denote the eigenvalues of $Y(T, s)$. By standard results concerning continuous dependence of solutions of differential equations on parameters and Rouche's theorem of complex analysis, the moduli $\left|\mu_{1}(s)\right|$ and $\left|\mu_{2}(s)\right|$ depend continuously on $s$. We shall show that

$$
\left|\mu_{j}(s)\right|<1, \quad j=1,2,0 \leq s \leq 1
$$

and, since $X(T)=Y(T, 1)$, this will prove the theorem.

By a well-known theorem of Liouville, for $0 \leq s \leq 1$,

$$
\mu_{1}(s) \mu_{2}(s)=\exp \int_{0}^{T} \operatorname{tr} B(t, s) d x=e^{-k T}<1 .
$$

For $s=0, B(s, t)$ is the constant matrix

$$
C=\left(\begin{array}{cc}
0 & 1 \\
-\gamma & -k
\end{array}\right)
$$

so $Y(t, 0)=e^{C t}$ and $\mu_{j}(0)=e^{\lambda_{j} T}, j=1,2$, where $\lambda_{1}$ and $\lambda_{2}$ are the eigenvalues of $C$. Since $k>0$ and $\gamma>0$, it is easy to compute that both $\lambda_{1}$ and $\lambda_{2}$ have a negative real part. Therefore, $\left|\mu_{j}(0)\right|<1$ for $j=1,2$.

If (13) were not true for $0 \leq s \leq 1$, then, by continuity, there would exist a number $s^{*}$ with $0<s^{*} \leq 1$ such that $\left|\mu_{j}\left(s^{*}\right)\right|=1$ for either $j=1$ or $j=2$.

The numbers $\mu_{1}(s)$ and $\mu_{2}\left(s^{*}\right)$ could not be complex conjugates, for otherwise $\left|\mu_{1}\left(s^{*}\right)\right|=\left|\mu_{2}\left(s^{*}\right)\right|=1$ contradicting (14). Therefore both $\mu_{1}\left(s^{*}\right)$ and $\mu_{2}\left(s^{*}\right)$ would be real, and so $\mu_{j}\left(s^{*}\right)= \pm 1$ for either $j=1$ or $j=2$. Since this contradicts the previously established fact that $Y(T, s)$ cannot have \pm 1 as an eigenvalue for all $s \in[0,1],(13)$ must hold and the theorem is proved.

Our main result now follows easily.

Theorem 2. Let $g(t, \xi)$ and $D_{2} g(t, \xi)$ be continuous for all $(t, \xi) \in \mathbf{R}^{2}$, let $g$ be $T$-periodic in $t$, and $k>0$ be constant. If there exist constants $a$ and $b$ such that (2) holds and such that there exists a closed disk $B$ in the complex plane centered at $\gamma=(a+b) / 2$ of radius $r>(b-a) / 2$ such that (9) holds, then (1) has a unique T-periodic solution which is locally, exponentially, asymptotically stable.

Proof. Since (9) obviously implies (3), there exists a unique $T$-periodic solution of (1)-call it $u_{0}(t)$. Since $\operatorname{col}\left(u_{0}(t), u_{0}^{\prime}(t)\right)$ is a $T$-periodic solution of the system

$$
\begin{aligned}
& u^{\prime}=v, \\
& v^{\prime}=-g(t, u),
\end{aligned}
$$

it follows from the well-known principle of linearized stability for periodic systems (see [3, p. 321-322]) that if the Floquet multipliers corresponding to this solution both have moduli less than 1 , then this solution is locally exponentially stable. Since the multipliers are the eigenvalues of the $2 \times 2$ matrix $X(T)$ where $X(t)$ is the matrix function defined by

$$
X^{\prime}(t)=A(t) X(t), \quad X(0)=I
$$


where

$$
A(t)=\left(\begin{array}{cc}
0 & 1 \\
-D_{2} g\left(t, u_{0}(t)\right) & -k
\end{array}\right)
$$

the assertion of the theorem follows from (2), (9), and Theorem 2.

We note that the conditions of the Theorem imply that there are no $2 T$ periodic solutions other than $u_{0}$.

We give specific bounds for $a$ and $b$ which extends the Loud-Leach condition. For simplicity we take $T=2 \pi$.

Theorem 3. Let $k>0$ and for each nonnegative integer $N$, let

$$
\begin{aligned}
& a_{N}(k)=\frac{k^{2}}{2}+N^{2}+N+\frac{1}{2}-\sqrt{\left(\frac{k^{2}}{2}+N+\frac{1}{2}\right)^{2}+k^{2} N^{2},} \\
& b_{N}(k)=\frac{k^{2}}{2}+N^{2}+N+\frac{1}{2}+\sqrt{\left(\frac{k^{2}}{2}+N+\frac{1}{2}\right)^{2}+k^{2} N^{2},} \\
& c_{N}(k)=\frac{k^{2}}{2}+\frac{N^{2}}{4}+\frac{N}{2}+\frac{1}{2}-\sqrt{\left(\frac{k^{2}}{2}+\frac{N}{2}+\frac{1}{2}\right)^{2}+\frac{(k N)^{2}}{4}}, \\
& d_{N}(k)=\frac{k^{2}}{2}+\frac{N^{2}}{4}+\frac{N}{2}+\frac{1}{2}+\sqrt{\left(\frac{k^{2}}{2}+\frac{N}{2}+\frac{1}{2}\right)^{2}+\frac{(k N)^{2}}{4}} .
\end{aligned}
$$

If $g$ and $D_{2} g$ are continuous, $g$ is $2 \pi$-periodic in $t$, and (2) holds where, for some $N \geq 0$,

$$
a_{N}(k)<a \leq b<b_{N}(k),
$$

then (1) has a unique $2 \pi$-periodic solution. If

$$
c_{N}(k) \leq a \leq b<d_{N}(k)
$$

and (2) holds then (1) has a unique $2 \pi$-periodic solution which is locally, exponentially, asymptotically stable.

Proof. We prove only the second statement, the proof of the first statement is entirely similar.

Let $N$ be a fixed nonnegative integer and let

$$
\begin{aligned}
s & =\frac{k^{2}}{2}+\frac{N^{2}}{4}+\frac{N}{2}+\frac{1}{2} \\
R & =\sqrt{\left(\frac{k^{2}}{2}+\frac{N}{2}+\frac{1}{2}\right)^{2}+\frac{(k N)^{2}}{4}} .
\end{aligned}
$$

The complex numbers

$$
\frac{m^{2}}{4}-\frac{m i k}{2}, \quad m=0, \pm 1, \pm 2, \ldots
$$


all lie on the parabola $k^{2} x=y^{2}$ in the complex plane. The point $s$ is at a distance $R$ from the points corresponding to the complex numbers $N^{2} / 4 \pm$ $N i k / 2$ and $(N+1)^{2} / 4 \pm(N+1) i k / 2$ and the open disk centered at radius $R$ does not contain any of the numbers (17). Since $c_{N}(k)=s-R$ and $d_{N}(k)=$ $s+R$, if (16) holds, then we can find a number $d$ such that

$$
s-R<s-d<a \leq b<s+d<s+R .
$$

Therefore, since the closed disk of radius $d$ centered at $s$ does not contain any of the numbers (17), the second assertion of the theorem follows from Theorem 2 .

Finally, we give a simple example which shows that if the conditions of the preliminary lemma hold but the conditions of Theorem 2 do not hold, then the unique periodic solution can be unstable.

Example. Consider the linear differential equation

$$
u^{\prime \prime}(t)+k u^{\prime}(t)+\frac{1}{4}[1+\varepsilon \cos t] u(t)=0
$$

where $k$ is small and positive $|\varepsilon|$ is small and $\varepsilon \neq 0$. It is clear that for $|\varepsilon|$ small, there exist numbers $a$ and $b$ such that the conditions of the preliminary lemma hold, so there exists a unique $2 \pi$-periodic solution; namely, $u \equiv 0$.

On the other hand, for all small $\varepsilon \neq 0$, the Floquet multipliers $\mu_{1}$ and $\mu_{2}$ corresponding to the trivial solution of

$$
u^{\prime \prime}(t)+\frac{1}{4}[1+\varepsilon \cos t] u(t)=0
$$

are real and satisfy

$$
\mu_{1}<-1<\mu_{2}<0 \text {. }
$$

(This is stated without proof in [1, p. 204-205].) In fact, if $y_{j}(t, \varepsilon), j=1,2$, are the solutions of (19) defined by the initial conditions $y_{1}(0, \varepsilon)=y_{2}^{\prime}(0, \varepsilon)=$ $1, y_{2}(0, \varepsilon)=y_{1}^{\prime}(0, \varepsilon)=0$, and $\Delta(\varepsilon)=y_{1}(2 \pi, \varepsilon)+y_{2}^{\prime}(2 \pi, \varepsilon)$, then $\mu_{1}$ and $\mu_{2}$ are the roots of the quadratic

$$
\mu^{2}-\Delta(\varepsilon) \mu+1=0 .
$$

Using the fact that $y_{k}(t, \varepsilon)$ are analytic in $\varepsilon$, a long but straightforward computation shows that

$$
\Delta(\varepsilon)=-2-\frac{\pi}{64} \varepsilon^{2}+O\left(\varepsilon^{3}\right)
$$

as $\varepsilon \rightarrow 0$.

Fix $\varepsilon \neq 0$ such that $(20)$ holds. Since the multipliers corresponding to the trivial solution of (18) are the eigenvalues of $X(2 \pi)$, where $X(t)$ is the fundamental matrix corresponding to (7), where $p(t)=\frac{1}{4}[1+\varepsilon \cos t]$, and for $k=0$ these are the numbers $\mu_{1}$ and $\mu_{2}$ in (20), it follows from the continuous dependence of solutions with respect to parameters, that for $k$ small 
and positive, the multipliers of the trivial solution of (18) will also satisfy the condition (20). Therefore, the solution will be unstable.

In [9] Ortega considers the problem

$$
u^{\prime \prime}+k u^{\prime}+f(t, u)-s=0,
$$

where $k>0, f$ and $D_{2} f(t, \xi)$ is strictly increasing in the second variable, $f$ is $T$-periodic in $t$, and $s$ is a real parameter. If there exist $\xi_{1}$ and $\xi_{2}$ such that $D_{2} f(t, \xi)<0$ for all $t$ if $\xi \leq \xi_{1}$ and $D_{2} f(t, \xi)>0$ for all $t$ if $\xi \geq \xi_{2}$, then the condition (9) can never be satisfied for $m=0$, where $g(t, \xi)=f(t, \xi)-s$. However, for this case, under the additional assumption that

$$
D_{2} f(t, \xi)<\pi^{2} / T^{2}+k^{2} / 4
$$

for all $(t, \xi) \in \mathbf{R}^{2}$, the results of [9] show that there exists $s_{0}$ such that (21) has a $T$-periodic solution if and only if $s \geq s_{0}$ and for $s>s_{0}$ there are exactly two $T$-periodic solutions, one of which is asymptotically stable and the other unstable.

We are grateful to the referee for calling our attention to the recent literature concerning (1) when $k \neq 0$. (See [12-16].)

\section{REFERENCES}

1. V. I. Arnold, Ordinary differential equations, Massachusetts Institute of Technology, Cambridge, 1973.

2. G. Borg, Uber die Stabilität gewisser Klassen von linearen differential gleichungen, Ark. Mat. Astr. Fys. 31 A (1944), 1-3.

3. E. A. Coddington and N. Levinson, Theory of ordinary differential equations, McGraw Hill, New York, 1955.

4. C. L. Dolph, Nonlinear integral equations of the Hammerstein type, Trans. Amer. Math. Soc. 66 (1949), 289-307.

5. D. E. Leach, On Poincaré's perturbation theorem and a theorem of W. S. Loud, J. Differential Equations 7 (1970), 34-53.

6. W. S. Loud, Periodic solutions of nonlinear differential equations of Duffing type, in Differential and Functional Equations, Proceedings of U.S.-Japan Seminar (W. A. Harris, Jr. and Y. Sibuya, eds.), New York, 1967, pp. 199-224.

7. W. Magnus and S. Winkler, Hill's equation, Interscience Publishers, New York, 1966.

8. J. Mawhin, Contractive mappings and periodic perturbed conservative systems, Arch. Math. (Brno) 12 (1976), 67-73.

9. R. Ortega, Stability and index of periodic solutions of an equation of Duffing type, Boll. Un. Mat. Ital. (7) (1989), 3-13.

10. S. Wallach, The stability of differential equations with periodic coefficients, Proc. Nat. Acad. Sci. U.S.A. 34 (1948), 203-204.

11. S. A. Williams, A nonlinear elliptic boundary value problem, Pacific J. Math. 44 (1973), 767-774.

12. P. Drabek and S. Invernizzi, On the periodic BVP for the forced duffing equation with jumping nonlinearity, Nonlinear Anal. 10 (1986), 643-650.

13. C. P. Gupta, J. M. Nieto, and L. Sanchez, Periodic solutions of some Leonard and Duffing equations, J. Math. Anal. Appl. 140 (1989), 67-82. 
14. P. Habets and G. Metzen, Existence of periodic solutions of Duffing equations, J. Differential Equations 78 (1989), 1-32.

15. R. Manasevich, A nonvariational version of a max-min principle, Nonlinear Anal. 7 (1983), 565-570.

16. R. Reissig, Contractive mappings and periodically perturbed non-conservative systems, Atti Accad. Naz. Lincei Rend. C. Sci. Fis. Mat. Natur. (8) 58 (1975), 696-702.

Department of Mathematics and Computer Science, University of Miami, Coral GABLES, FLORIDA 33124

Department of Mathematics, University of Connecticut, Storrs, Connecticut 06268 